\title{
Acute haemoabdomen associated with Angiostrongylus vasorum infection in a dog: A case report
}

\author{
Willesen, J.L.,Bjornvad, C.R. and Koch, J. \\ Department of Small Animal Clinical Sciences, Faculty of Life Sciences, University of Copenhagen, Dyrlaegevej \\ 16, 1870 Frederiksberg C, Denmark
}

\begin{abstract}
A one-year-old intact female, Danish shorthaired pointer was referred to the emergency service with a history of acute collapse and pale mucous membranes after a month of reduced activity but with no other clinical signs. An ultrasound examination of the abdomen indicated the presence of a large amount of free fluid with no obvious cause such as neoplasia or splenic rupture. Fluid analysis had the macroscopic appearance of blood with no signs of infection or neoplasia. Multiple Angiostrongylus vasorum L1 larvae were revealed on a direct rectal faecal smear. The dog was treated with fenbendazole $25 \mathrm{mg} / \mathrm{kg}$ orally once daily for 20 days and given supportive treatment. The dog was stabilised on this treatment. Haemoabdomen is a clinical sign where surgical intervention is often considered an integral part of the diagnostic investigation (i.e., laparotomy) or treatment. Failing to make the diagnosis of canine angiostrongylosis before performing surgery may have a serious adverse affect on the outcome. Consequently, in areas where $A$. vasorum is enzootic, a Baermann test and a direct faecal smear should be included in the initial diagnostic investigation of all dogs presenting with bleeding disorders of unknown origin.
\end{abstract}

Keywords: Angiostrongylus vasorum, coagulopathy, haemoabdomen, treatment

\section{Corresponding author:}

Jakon L Willesen

Department of Small Animal Clinical Sciences,

Faculty of Life Sciences, University of Copenhagen,

Dyrlaegevej 16, 1870 Frederiksberg C, Denmark

Email: jwi@life.ku.dk

Phone: + 4535332955

Fax: + 4535332929

Irish Veterinary Journal Volume 61 Number 9 591-593, 2008

Angiostrongylus vasorum (French heartworm) is a nematode causing verminous pneumonia in dogs. Dogs and other Canidae such as foxes serve as definitive hosts, while snails and slugs act as intermediate hosts. It has a worldwide distribution and in Europe it is considered enzootic in certain areas in France, Denmark, Ireland and the UK (Bourdeau, 1993; Brennan et al., 2004; Chapman et al., 2004; Koch and Willesen, 2008). The adult metastrongyle (13-21 $\mathrm{mm})$ resides in the pulmonary arteries and the right side of the heart (Rosen et al., 1970). First-stage (L1) larvae migrate through the respiratory system via the trachea to the gastrointestinal-tract and are excreted in faeces (Bolt et al., 1994). The migration of the L1 larvae through the blood-air barrier and the subsequent verminous pneumonia and pulmonary thrombosis causes the clinical signs (i.e., cough, exercise intolerance, dyspnoea) that are commonly associated with the pulmonary system (Martin et al., 1993; Brennan et al., 2004; Chapman et al., 2004). Haematological changes such as neutrophilia, thrombocytopaenia and anaemia have been reported (Chapman et al., 2004) whereas the most frequent changes in biochemistry are increased globulin levels and decreased fructosamine (Chapman $e t$ al., 2004; Willesen et al., 2006). A fatal outcome of infection is often associated with coagulation disorders. Suggested mechanisms behind the coagulopathy are induced immunemediated thrombocytopaenia, disseminated intravascular coagulation (DIC) or secretion of anticoagulants by the adult worms (Schelling et al., 1986; Ramsey et al., 1996; Gould and McInnes, 1999; Cury et al., 2002; Whitley et al., 2005). Clinical presentation of associated bleeding disorders may vary between petechial or ecchymotic bleeding on mucosal surfaces, dermis or sclera to intracranial or CNS bleeding (Cury and Lima, 1996; Brennan et al., 2004; Chapman et al., 2004; Garosi et al., 2005; Wessmann et al., 2006). To the authors' knowledge, this is the first reported case of haemoabdomen associated with an $A$. vasorum infection. A one-year-old intact female Danish shorthaired pointer (body weight $25 \mathrm{~kg}$ ) was referred to the emergency service at the Department of Small Animal Clinical Sciences at the University of Coprnhagen with a history of acute collapse and pale mucous membranes. The owner had noted the dog to be less active over the past month, but with no other clinical signs more commonly related to canine angiostrongylosis such as cough, dyspnoea or exercise intolerance. The dog presented with a temperature of $37.1^{\circ} \mathrm{C}$, pulse $120 / \mathrm{min}$, respiration rate of $28 / \mathrm{min}$ and pale pink, dry mucous membranes with a prolonged capillary refill time greater than two seconds. A single petechia was 


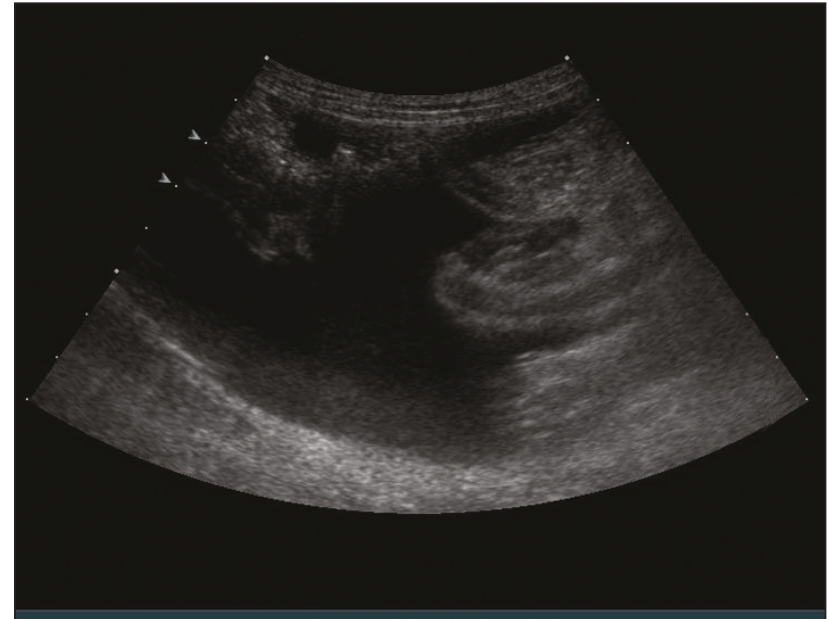

Figure 1: Abdominal ultrasound of a dog diagnosed with $\boldsymbol{A}$. vasorum. A large amoun of free intraabdominal fluid was observed. PCV was $35 \%$ in a sample obtained by abdominocentesis. No signs of neoplasia or infection were observed.

present on the gingival mucosa. The most predominant changes in haematological and biochemical profiles were neutrophilia, packed cell volume (PCV) of 24\%, elevated urea and creatinine and reduced fructosamine (Table $\mathbf{1}$ ).

Table 1. Haematological, biochemical and coagulation parameters at time of diagnosis

\begin{tabular}{|c|c|c|c|}
\hline Haematological parameters & (Reference range) & Measurement & Changes \\
\hline Packed cell volume (PCV) & $0.39-0.55 \mathrm{~L} / \mathrm{L}$ & 0.24 & $\downarrow$ \\
\hline Total red blood cells (RBC) & $4.6-8.4 \times 109 / \mathrm{L}$ & 3.4 & $\downarrow$ \\
\hline Platelets & $200-500 \times 109 / L$ & $90 *$ & \\
\hline Total white blood cells (WBC) & $6.5-18.1 \times 109 / \mathrm{L}$ & 26.8 & $\uparrow$ \\
\hline Segmented neutrophils & $3.2-12.1 \times 109 / L$ & 22.0 & $\uparrow$ \\
\hline Lymphocytes & $1.0-4.8 \times 109 / \mathrm{L}$ & 3.1 & \\
\hline Monocytes & $0.0-1.2 \times 109 / \mathrm{L}$ & 1.6 & $\uparrow$ \\
\hline Eosinophils & $0.0-1.2 \times 109 / \mathrm{L}$ & 0.02 & \\
\hline \multicolumn{4}{|l|}{ Biochemical parameters } \\
\hline Alanine aminotransferase (ALAT) & $6-120 \mathrm{U} / \mathrm{L}$ & 124 & $\uparrow$ \\
\hline Alkaline phosphatiase (BASP) & 19.8-174 U/L & 109 & \\
\hline Glucose & 3.9-6.55 mmol/L & 9.24 & $\uparrow$ \\
\hline Urea & $3.3-9.4 \mathrm{mmol} / \mathrm{L}$ & 17.3 & $\uparrow$ \\
\hline Creatinine & $44-130 \mathrm{mmol} / \mathrm{L}$ & 179.2 & $\uparrow$ \\
\hline Amylase & $186-798 \mathrm{U} / \mathrm{L}$ & 1706 & $\uparrow$ \\
\hline Lipase & $6-498 \mathrm{U} / \mathrm{L}$ & 217.4 & \\
\hline Cholesterol & $3.5-6.99 \mathrm{mmol} / \mathrm{L}$ & 2.5 & $\downarrow$ \\
\hline Bilirubin & $1.0-5.0 \mu \mu \mathrm{mol} / \mathrm{L}$ & 4.0 & \\
\hline Albumin & $26.0-44-0 \mathrm{~g} / \mathrm{L}$ & 26.8 & \\
\hline Protein & $57.0-82.0 \mathrm{~g} / \mathrm{L}$ & 53.1 & $\downarrow$ \\
\hline Fructosamine & 235-344 $\mu \mu \mathrm{mol} / \mathrm{L}$ & 212 & $\downarrow$ \\
\hline Bile acid & 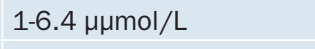 & 1 & \\
\hline Calcium & $2.2-3.3 \mathrm{mmol} / \mathrm{L}$ & 3.2 & \\
\hline Magnesium & $0.63-1.05 \mathrm{mmol} / \mathrm{L}$ & 0.94 & \\
\hline Phosphate & 0.91-1.96 mmol/L & 2.47 & $\uparrow$ \\
\hline Potassium & $3.81-5.07 \mathrm{mmol} / \mathrm{L}$ & 3.8 & $\downarrow$ \\
\hline Sodium & $142.2-153.6 \mathrm{mmol} / \mathrm{L}$ & 141.9 & $\downarrow$ \\
\hline \multicolumn{4}{|l|}{ Coagulation parameters } \\
\hline Fibrinogen & $(1.0-4.0 \mathrm{~g} / \mathrm{L})$ pool 0.64 & 0.05 & \\
\hline Prothrombin time (PT) & $($ pool $8.2 \mathrm{sec})$ & 19.2 & $\uparrow$ \\
\hline Activated prothrombin time (APPt) & (pool $18.5 \mathrm{sec})$ & 15.0 & \\
\hline D-dimer & (0.0-0.5 mg/L) pool 0.1 & 3.2 & $\uparrow$ \\
\hline
\end{tabular}

* aggregates seen on blood smear at 40x magnification.
An ultrasound examination of the abdomen (Figure 1)

suggested a large amount of free fluid with no obvious cause such as neoplasia or splenic rupture. Abdominocentesis was performed and the aspirate had the macroscopic appearance of blood, a PCV of $35 \%$ and

no signs of infection or neoplasia on a cytological examination. The coagulation profile showed prolonged Prothrombin time (PT) and increased D-dimer (Table 1). Phytomenadion (Konakion, Roche A/S, Hvidovre,

Denmark) treatment was initiated due to potential coumarin poisoning and for initial stabilisation, the dog received two transfusions; one unit of full blood $(18 \mathrm{ml} / \mathrm{kg})$ and one unit of fresh frozen plasma $(9 \mathrm{ml} / \mathrm{kg})$. A PCV of $24 \%$ is not usually a clear indication for a blood transfusion. However, in the present case, the authors chose to carry out the procedure as a continuing loss of blood could not be ruled out at this stage. Urinalysis of a voided sample was unremarkable except that a single $A$. vasorum L1 larva was detected on microscopy. Subsequently, multiple L1 larvae were revealed using a direct faecal smear technique (Willesen et al., 2004). Due to the rapid establishment of the diagnosis, a buccal mucosal bleeding test was not performed. The dog was treated with fenbendazole $25 \mathrm{mg} / \mathrm{kg}$ orally once daily for 20 days following a protocol known to be efficient in the treatment of $A$. vasorum infections (Willesen et al., 2007). To avoid possible anaphylactic reactions, prednisolone acetate at $1 \mathrm{mg} / \mathrm{kg}$ (Prednisolon DAK, Nycomed, Roskilde, Denmark) was administered once daily for 12 days. The dog stabilised on this treatment and was discharged after two days of hospitalisation and monitoring. Four weeks later, a complete resolution of clinical signs and normalisation of haematological and coagulation blood parameters were found. Haemoabdomen associated with canine angiostrongylosis has not been reported before, although spontaneous bleeding at other sites has been reported. In the present case, no history of either surgical or accidental trauma existed. Neoplasia could not be demonstrated and treatment with phytomenadion was initially started in case of a rodenticide poisoning, although the owner reported it unlikely that the dog had been in contact with such compounds. Coumarin poisoning is not usually associated with neutrophilia and 
decreased fructosamine, as in this case, but could account for the prolonged PT. Prolonged PT is also often reported in dogs infected with A. vasorum (Ramsey et al., 1996; Cury et al., 2002). Increased D-dimer indicates formation of thrombi, but is non-specific regarding the underlying cause. Prolonged PT and elevated D-dimer could be indicative of disseminated intravascular coagulation (DIC) as is often reported in cases of canine angiostrongylosis (Ramsey et al., 1996; Garosi et al., 2005). Immune-mediated thrombocytopaenia has been reported in association with A. vasorum infection (Gould and McInnes, 1999). However, with a platelet count of $90 \times 10 \% / \mathrm{L}$ and aggregates present on the blood smear, it was considered unlikely to have caused the bleeding, since bleeding caused by low platelet number usually occurs only with platelet count below $40 \times 10 \%$ L. The acute onset of clinical signs might also have been caused by a ruptured small vessel. Aberrant migration of adult worms probably occurs frequently (Oliveira-Junior $\mathrm{et} \mathrm{al}$., 2004) and rupture of the femoral artery has been reported previously in a case report of an experimentally infected dog (Cury and Lima, 1996). Finally, the possible role of the parasites secreting an anticoagulant may contribute to the development of bleeding problems (Cury et al., 2002) This mechanism, however, needs to be further investigated. The finding of L1 larvae in the urinalysis may be explained by ectopic L1 larvae (Oliveira-Junior et al., 2004) or faecal contamination of the voided urine sample. However, due to the presumed hypocoagulable state of the dog, sampling by cystocentesis was not performed.

Haemoabdomen is a clinical sign where surgical intervention is often considered an integral part of the diagnostic investigation (i.e., laparotomy) or treatment. Failing to make the diagnosis of canine angiostrongylosis before performing surgery may have an adverse affect on the outcome for the patient. In conclusion, in areas where $A$. vasorum is enzootic, a Baermann test and a direct faecal smear should be included in the initial diagnostic investigation of all dogs presenting with bleeding disorders of unknown origin.

\section{Acknowledgements}

Dr. James Miles is thanked for the English review of this paper.

\section{References}

Bolt, G., Monrad, J., Koch, J. and Jensen, A.L. (1994). Canine Angiostrongylosis - A Review. Veterinary Record 135: 447-452. Bourdeau, P. (1993). Canine Angiostrongylus-Vasorum Infestation. Recueil de Medecine Veterinaire 169: 401-407.

Brennan, S.F., McCarthy, G., McAllister, H., Bassett, H., and Jones,

B.R. (2004). Clinical signs, diagnosis and treatment of three dogs with angiostrongylosis in Ireland. Irish Veterinary Journal 57: 103-109. Chapman, P.S., Boag, A.K., Guitian, J. and Boswood, A. (2004).

Angiostrongylus vasorum infection in 23 dogs (1999-2002). Journal of Small Animal Pracice 45: 435-440.

Cury, M.C. and Lima, W.S. (1996). Rupture of femoral artery in a dog infected with Angiostrongylus vasorum. Veterinary Parasitology 65: 313-315. Cury, M.C., Lima, W.S., Guimaraes, M.P. and Carvalho, M.G. (2002).

Hematological and coagulation profiles in dogs experimentally infected with Angiostrongylus vasorum (Baillet, 1866). Veterinary Parasitology 104: 139-149.

Garosi, L.S., Platt, S.R., McConnell, J.F., Wrayt, J.D. and Smith, K.C. (2005). Intracranial haemorrhage associated with Angiostrongylus vasorum infection in three dogs. Journal of Small Animal Practice 46: 93-99.

Gould, S.M. and McInnes, E.L. (1999). Immune-mediated

thrombocytopaenia associated with Angiostrongylus vasorum infection in a dog. Journal of Small Animal Practice 40: 227-232.

Koch, J. and Willesen, J.L. (2008). Canine pulmonary angiostrongylosis - an update. The Veterinary Journal "In previous."

Martin, M.W.S., Ashton, G., Simpson, V.R. and Neal, C. (1993).

Angiostrongylosis in Cornwall - Clinical Presentations of Eight Cases. Journal of Small Animal Practice 34: 20-25.

Oliveira-Junior, S.D., Barcante, J.M.P., Barcante, T.A., Ribeiro, V.M. and Lima, W.S. (2004). Ectopic location of adult worms and first-stage larvae of Angiostrongylus vasorum in an infected dog. Veterinary Parasitology 121 : 293-296.

Ramsey, I.K., Littlewood, J.D., Dunn, J.K. and Herrtage, M.E. (1996).

Role of chronic disseminated intravascular coagulation in a case of canine angiostrongylosis. Veterinary Record 138: 360-363.

Rosen, L., Ash, L.R., and Wallace, G.D. (1970). Life history of canine lungworm Angiostrongylus vasorum (Baillet). American Journal of Veterinary Research 31: 131-141.

Schelling, C.G., Greene, C.E., Prestwood, A.K. and Tsang, V.C.W. (1986). Coagulation abnormalities associated with acute Angiostrongylus vasorum infection in dogs. American Journal of Veterinary Research 47: 2669-2673.

Wessmann, A., Lu, D., Lamb, C.R., Smyth, B., Mantis, P., Chandler, K., Boag, A., Cherubini, G.B. and Cappello, R. (2006). Brain and spinal cord haemorrhages associated with Angiostrongylus vasorum infection in four dogs. The Veterinary Record 158: 858-863.

Whitley, N.T., Corzo-Menendez, N., Carmichael, N.G. and McGarry, J.W. (2005). Cerebral and conjunctival haemorrhages associated with von Willebrand factor deficiency and canine angiostrongylosis. Journal of Small Animal Practice 46: 75-78.

Willesen, J., Møller, J., Koch, J., Jensen, A.L. and Kristensen, A.T. (2004).

Tidlig diagnostik af Angiostrongylus vasorum (Fransk hjerteorm) og Crenosoma vulpis (Rævens lungeorm) hos hunde er mulig ved hjælp af modificeret Baermann test. Dansk Veterincer Tidsskrift 87: 6-10. (English summary: Early diagnosis of Angiostrongylus vasorum (French Heartworm) and Crenosoma vulpis (Fox lungworm) is possible by means of modified Baermann Test).

Willesen, J.L., Jensen, A.L., Kristensen, A.T., Kjelgaard-Hansen, M., Jessen, R. and Koch, J. (2006). Serum fructosamine concentrations in 59 dogs naturally infected with Angiostrongylus vasorum. Journal of Veterinary Medicine Series A 53: 266-269.

Willesen, J.L., Kristensen, A.T., Jensen, A.L., Heine, J. and Koch, J. (2007). Efficacy and safety of imidacloprid/moxidectin spot-on solution and fenbendazole in the treatment of dogs naturally infected with Angiostrongylus vasorum (Baillet, 1866). Veterinary Parasitology 147: 258-264. 